\title{
Is awareness of obesity and type 2 diabetes from physical inactivity in the home and workplace well recognized?
}

\begin{abstract}
Most recommendations from governmental agencies and the American Heart Association have focused on daily exercise regimens as a means to achieve good cardiovascular health and diminish risk of premature mortality. It has recently become apparent that physical inactivity marked by excessive sitting and/or lying down is a major risk for the worldwide growing epidemic of obesity and type 2 diabetes. This epidemic cannot be addressed by short term exercise if the rest of the day is spent in physical inactivity. Even when recognized, interventions to limit sitting time have failed to achieve major success because of the strong behavioral affinity to sit. In this paper, we propose that physical inactivity can be tolerated if an effortless, simulated jogging device that does not require multitasking is used at least two hours daily. Since watching television and/or a computer screen takes up several hours daily for most individuals, this recommendation is easily accomplished during such activities.
\end{abstract}

Volume 6 Issue 5 - 2017

\author{
Marvin A Sackner,' Jose A Adams ${ }^{2}$ \\ 'Sackner Wellness Products LLC, USA \\ ${ }^{2}$ Mt Sinai Medical Center, USA
}

Correspondence: Marvin A Sackner, Sackner Wellness Products LLC, 555 NE 34th Street, PHI, Miami FL 33।37, USA, Tel 30533388II,Email Artchive@msn.com

Received: April 13, 2017 | Published: May 09, 2017
Abbreviations: BDNF, brain derived neurotrophic factor; LTPA, lower leisure time physical activity; NO, nitric oxide

\section{Introduction}

For over 15years, excessive sitting has been identified as deleterious to health because it increases risk of obesity, cardiovascular disease, Type 2 diabetes, metabolic syndrome, dementia, colon, breast and prostate cancers as well as overall mortality. With widespread introduction of computers into the workplace and increased uninterrupted periods of sitting, workers spend a great deal of their work-time viewing a computer screen. At home, they spend a significant amount of time sitting and watching television and dining.

Higher household income (both genders) and professional/ managerial occupations (men only) correlates to a high work related sitting time. Health-related factors (lower leisure time physical activity [LTPA], higher BMI - men, and higher energy consumption women) are associated with elevated levels of both work-related and $\mathrm{TV}$ viewing time. Targeted interventions to reduce sitting time should be available to all but prioritized to high income, obese, managerial individuals who spend a great deal of their time watching television and computer screens.

Prolonged sitting is accompanied by varying degrees of arteriosclerosis, vascular stiffening, Type 2 diabetes, obesity and endothelial dysfunction (vide infra). The health hazards of excessive sitting for all-cause mortality and risk of cardiovascular disease are diminished either by walking at a leisurely 10,000 steps per day or brisk walking or jogging 30minutes a day (American Heart Association [AHA] recommendations). Jogging significantly lowers risk to cardiovascular mortality in contrast to walking 10,000 steps per day that has a much lesser effect. Most elderly Americans fail to reach AHA goals of exercise that are beneficial for cardiovascular health. Even increasing the daily recommendation of physical exercise for 30 minutes to 60 and 75 minutes a day cannot compensate for the negative effects of physical inactivity on increasing insulin resistance and plasma lipid levels if the rest of the day is spent sitting.
Substituting six hours of sitting with four hours of walking and two hours of standing has more health benefits than one hour of physical exercise when energy expenditure is kept constant. However, such a regimen is unsustainable for most of the population and another solution is the purpose of this presentation.

\section{Endothelial function}

The major reasons for the benefits of increased physical activity and diminished physical inactivity, each individual risk factor, relate to their capability to preserve and/or improve endothelial function. The endothelium is the inner lining layer of blood vessels which when mechanically stimulated by increased shear stress (friction) brought about by increased blood flow and/or increased number of pulses from the rapid heartbeat of exercise or a passive simulated jogging device (Sackner Wellness Products LLC, Miami, FL) increase release of several beneficial substances into the circulation. These substances include among others nitric oxide (NO) which has major anti-arteriosclerotic, anti-inflammatory and antioxidant actions, Brain Derived Neurotrophic Factor (BDNF) that promotes neural repair and improved cognition and SIRT1 that has anti-aging properties. Blood flow stagnates during excessive sitting and bed rest leading to nitric oxide and BDNF deficiencies associated with a higher risk to coronary artery disease and mortality as well as increased risk to dementias. ${ }^{1-5}$

American adults and children spend more than half their waking time sitting. This produces impaired insulin sensitivity, metabolic function and endothelial function, which are classic markers of arteriosclerotic heart disease (ASHD). Sitting reduces muscular activity of the lower extremities which decreases leg blood flow, increases blood pooling in the calf, augments mean arterial pressure, and deforms arterial segments resulting in low mean shear stress (SS). The latter activates distinct physiological mechanisms which appear to be protective against ASHD; specifically, through a SS-induced endothelium-derived nitric oxide mechanism. Reduced bioavailability of nitric oxide creates a pro-oxidant milieu resulting in increased oxidative stress that further attenuates endothelial function. 


\section{Fidgeting}

Uninterrupted sitting causes an abrupt and significant decline in blood flow to the legs. When blood flow drops, there is reduced friction (shear stress) to the cells lining the inner walls (endothelium) of blood vessels. With reduced friction, these cells pump out substances that contribute over time to hardening and narrowing of the arteries. Researchers measured the level of normal blood flow through one of the main arteries in their legs and determined how well that artery responded to changes in blood pressure - a marker of arterial health. Most importantly, volunteers in this study were asked to keep one leg perfectly still, the foot flat against the floor and unmoving. With the other leg, the volunteers were told to fidget - tapping their feet against the ground for one minute about 250 times per minute and then staying still for four minutes over a three-hour period.

Blood flow in the unmoving leg fell but rose in the fidgeting leg, compared both to baseline levels and to the unmoving leg. At the end of the three hours, the vessel in the unmoving leg responded poorly to changes in blood pressure as it had during baseline testing. But the artery in the fidgeting leg responded as well as or better than it had at baseline to changes in blood pressure. The ideal goal of continuous fidgeting while sitting over minutes or hours is virtually unobtainable in the general population because of the rapid onset of muscle fatigue. In this paper, we describe a passive simulated jogging device that can rapidly tap the feet on motorized pedals effortlessly and repetitively against a semi-rigid at rigid surface at speeds from about 120 to 200 steps per minute (vide infra). This tapping motion serves as an Automated Fidgeting Machine to produce increased pulsatile shear stress leading to healthy blood vessels, thereby mitigating the adverse consequences of sitting time

\section{Obesity \& diabetes epidemic}

In the United States, greater than $33 \%$ of adults and $17 \%$ of children are obese which has led to a dramatic increase in prediabetes. Current estimates indicate that one-third of American adults meet the criteria for prediabetes, and, in addition to type 2 diabetes (T2D) with obesity is closely associated with comorbidities such as cardiovascular disease, hypertension, atherosclerosis, stroke, and cancer. The principal cause of obesity is energy imbalance, e.g., the calories consumed are greater than those utilized by bodily processes. The average consumption of calories in the United States has increased by $>200 \mathrm{kcal} /$ day per person in recent years, which is partly attributable to the abundance of affordable, widely marketed, energy-dense foods. Nevertheless, the balance between caloric intake and energy expenditure is complex and regulated by many factors, most notably endothelial derived nitric oxide that is increased by shear stress to the endothelium but reduced by physical inactivity.

Endothelial dysfunction induced by obesity is an important risk factor that impairs blood flow to various organs. Obesity induced endothelial dysfunction is associated with decreased eNO production due to impaired endothelial NO synthase activity and expression as well as increased production of superoxide anion and the endogenous eNO inhibitor ADMA, together with increased vasoconstrictor factors, such as endothelin-1 and sympathetic nerve activation. Insulin resistance and Type 2 diabetes augment obesity-induced endothelial dysfunction.

Endothelial derived nitric oxide (eNO) has both antiobesogenic and insulin-sensitizing properties. Its antiobesogenic role relates to its capability of increasing fat oxidation in peripheral tissues such as skeletal muscle, liver, and adipose tissue as well as decreasing lipid synthesis in the liver. The impact of eNO on glucose metabolism and insulin sensitivity is underpinned by its capacity to increase transport of insulin and glucose to key peripheral tissues such as skeletal muscle and to regulate gluconeogenesis. Additionally, there may be implications for eNO-mediated hepatic insulin-sensitizing substance release, which enhances the vasodilatory properties of insulin. In addition, eNO appears to modulate insulin release. Therefore, eNO is one of the most critical regulatory components of metabolism, body composition, and insulin sensitivity. Despite solid evidence that increasing physical activity and aerobic exercise as well as reducing sitting time are important for maintaining good health, wellness programs for Americans who are

a) Office workers

b) Homebound

c) Work solely or mostly at home

d) Watch television at home as a major leisure activity

e) Deconditioned as post-hospital discharged patients, have not been well addressed.

\section{Office workers}

Employed, white collar workers spend about $33 \%$ to $65 \%$ of their workday sitting and became the first defined group of individuals to test interventions for limiting "sitting disease." These work-time interventions include intermittent standing from sitting posture in response to counselling, standing desks, computer prompted commands to stand or walk, treadmill desks that involved walking between 1-2 miles per hour, active stepping devices, and cycling devices. Most are associated with varying commitments of multitasking that are accompanied by declines in cognition and typing skills. Significant short-term health benefits (less than one year observations) have not been demonstrated. Indeed, for sitting-standing desks, after an initial period of enthusiasm for standing, this behavior is significantly diminished over time because Americans like to sit.

In a prospective, seven-year study of 155,000 elderly adults, replacing one hour of sitting per day with an equal amount of physical activity for less active individuals, was associated with lower risk to cardiovascular mortality and all-cause mortality. For more active individuals, replacement of sitting time with exercise but not for physical activity was associated with lower cardiovascular mortality. In a theoretical epidemiologic evaluation, replacement of two hours daily sitting time with standing or stepping led to lowering of cardiometabolic risk biomarkers such as cholesterol, triglycerides, etc. over a non-specified time-period. ${ }^{6-14}$

\section{Homebound}

In 2011, the prevalence of the homebound was about $6 \%$ including about 400,000 people who were completely homebound and $1,600,000$ who were mostly homebound. Among the semihomebound, the prevalence of those who never left home without personal assistance was 3\% and the prevalence of those who required help and/or had difficulty leaving home was $12 \%$. Only $12 \%$ of completely homebound individuals received primary care services at home.

The number of persons aged 65 and older in the United States has grown in the past century from 3 million ( $4 \%$ of population) to 
more than 39 million ( $13 \%$ of population) in 2008 . By 2030 , because of aging "baby boomers" and longer life expectancy, approximately 72 million, or more than one in five Americans, will be aged 65 and older. As the population ages, many will become homebound and need home-based care.

In recent years, hypertension (74\%), type 2 diabetes $(31 \%)$, and heart disease $(20 \%)$ were the three main physical problems for older adults being homebound. For older adults confined to a bed or chair, $22 \%$ had cognitive impairment and $2 \%$ suffered from depression. In 2004 , home health care costs amounted to $\$ 43$ billion, in 2009, $\$ 72$ billion, in 2014 \$104 billion, and in 2019 projected to reach $\$ 154$ billion. In $2000,79 \%$ of the elderly population with long-term care needs used home health care, costing $\$ 64$ billion, and $21 \%$ of this elderly population used nursing home care, costing \$138 billion. The current homecare system in the United States needs improvement Heart disease, hypertension, type 2 diabetes, dementia, and depression are the main health conditions contributing to the need for homecare in older adults. All can benefit by increased physical activity administered by nurses, physical therapists, caregivers and passive exercise devices.

\section{Home-based workers}

The percentage of workers whose work week was based at home increased from $3.6 \%$ to $4.3 \%$ of the population between 2005 and 2010 or between 4.8 million to 5.8 million workers. About 1 in 10 workers who worked exclusively from home were over the age of 65 in 2010. About one-fourth of home-based workers were in management, business, and financial occupations. Nearly half of home-basedworkers were self-employed. Thus, there is probably large numbers of home-based workers who are not reached by wellness programs and need programs targeted for self-starters. Home Viewers of Television. Americans sit and view television an average of five hours daily which also correlates with the time sitting at work. For example, if a worker sits 5hours at work, he is likely to sit 5hours at home.

Post-hospital discharge deconditioned patients. Nearly one fifth of Medicare patients discharged from a hospital - approximately 2.6million seniors - develop an acute medical problem within the subsequent 30 days that necessitates another hospitalization. Among patients admitted for treatment of heart failure, pneumonia, or chronic obstructive pulmonary disease (COPD), the cause of readmission is the same as that of the initial admission in only about $34 \%$. The remaining $66 \%$ of readmissions relate to "deconditioning' because patients are commonly deprived of sleep, disruption of normal circadian rhythms, nourished poorly, suffer pain and other discomforts, confront a baffling array of mentally challenging situations, receive medications that can alter cognition and physical function, and are confined almost exclusively to bed rest and/or a seated posture. Hospitalized patients commonly become deconditioned defined as "too much rest" that is accompanied by rapid loss of muscle mass. Such recently discharged patients often have impaired stamina, coordination, and strength as well as balance disorders, which place them at greater risk for accidents, falls, and infections, etc. Left untreated, the deconditioned state may last for weeks, months, or even years.

\section{Physical activity recommendations}

As adults age, they usually walk between 80 and 90 steps per minute. Wellness authorities have recommended that adults should walk 10,000 steps daily or about 2 hours daily to gain optimal health benefits. This has given rise to widespread marketing of wearable pedometers and accelerometers to measure step counts. But only a small fraction of the adult population can achieve this total time commitment of 2 hours daily by pleasurably walking. Current interventions in the workplace and the home on average usually fail to address this issue. For physical activity services, managers cite insufficient financial resources, staffing, and lack of specific physical activity service specifications as the main barriers.

\section{Passive exercise}

Over the past two decades, scientific publications have confirmed that repetitive, passive movements of the ankles or legs can produce the same physiological, direct and remote effects as active movements of aerobic exercise albeit to a lesser intensity. Devices to implement passive movements include passive/assisted motorized stationary bicycles that range in price from about $\$ 100$ to $\$ 5,000$. None of them operate readily with the feet under a standard desk. The less expensive free-standing devices weighing about $6.5 \mathrm{Kg}$, slide away from the user during their operation requiring bolting them to a plate or the floor thereby negating their portability. The more expensive devices weighing over a hundred pounds have a large footprint, are noisy, and not portable.

\section{Passive simulated jogging device}

Theoretically, an ideal passive exercise device (Figure 1 \& 2 ) should emulate features attributable to active exercise such as jogging or running without attempting to change the strongly ingrained habits of Americans viewing television and/or computer screens for hours in the sitting or supine postures as well as not producing a distraction. Such a patented, passive simulated jogging device (Gentle Jogger ${ }^{\circledR}$ Sackner Wellness Products, LLC, Miami, FL) has been tested over the past four years and will be introduced into the marketplace in the last half of 2017. It is a motorized, small footprint portable wellness device that rests on the floor in front of a chair or sofa. With feet placed upon pedals that alternate up and down, the ankles are reciprocally repetitively flexed and extended while the soles placed on pedals tap against a bumper within the device's chassis at the completion of the downward stroke. The device can also be strapped to the foot-end plate of a bed for individuals lying in bed. Tapping simulates striking the ground as in actual jogging, and with each strike a small pulse is added to the circulation thereby increasing friction to the inner lining of blood vessels known as pulsatile shear stress. The latter stimulates increased release of beneficial substances including endothelial derived nitric oxide into the circulation. Increased nitric oxide reduces insulin resistance and facilitates glucose uptake in skeletal muscle, features promoting prevention of Type 2 diabetes.

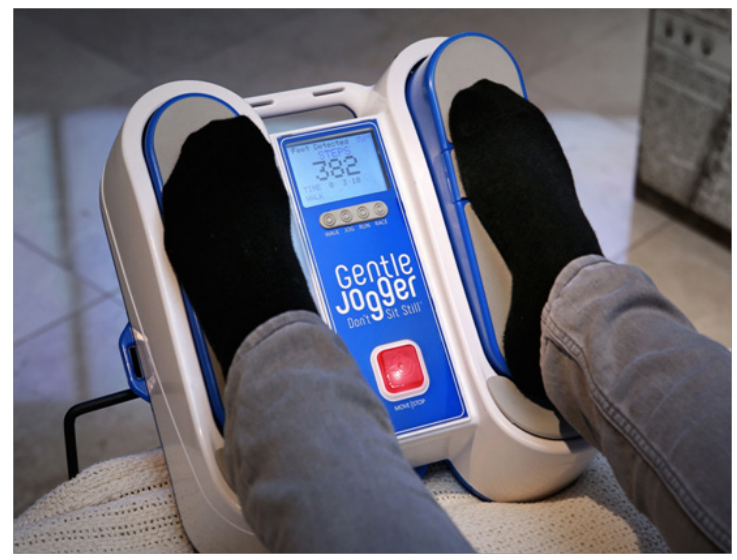

Figure I This depicts the top of passive simulated jogging device in the lying down posture with the device resting on the footplate of the bed. 


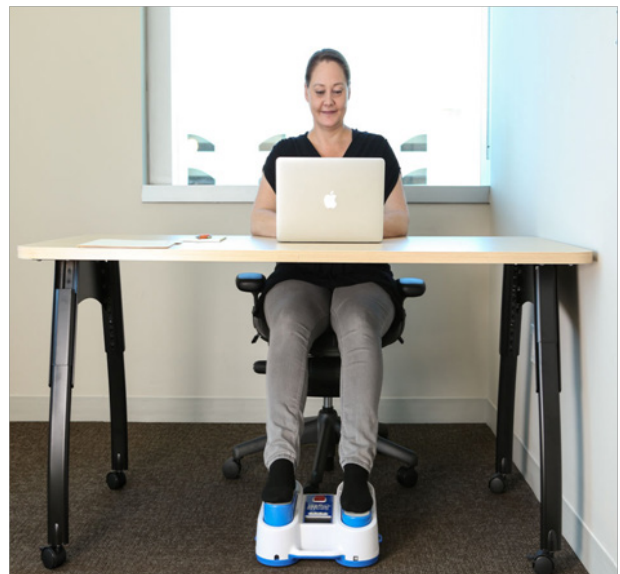

Figure 2 This depicts a typist with the passive sumulated jogging device placed underneath her desk.

Passive "simulated jogging" movements are associated with electromyographic signals to the thigh and lower body muscles but actual energy expenditure as measured with a MedGem ${ }^{\circledR}$ indirect calorimeter was minimal, e.g., slightly below standing energy expenditure which would not produce significant short-term weight loss. As shown in Figure 3, fall of skin temperatures as measured from thermal imaging over the legs and shoulders is consistent with the trend albeit to a lesser extent during active graded exercise because blood shifts from the skin to skeletal muscle. Passive movements of the ankles produce such systemic effects probably related to release of nitric oxide into the circulation (unpublished observations).
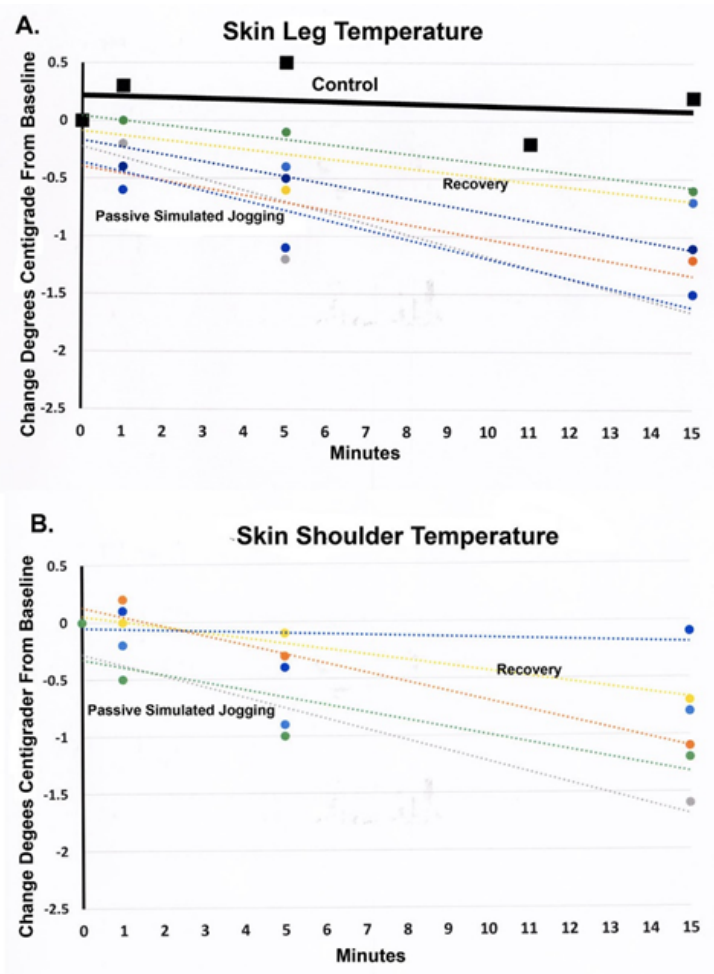

Figure 3 This depicts skin temperatures obtained with thermal imaging. Panel A.Skin temperatures of the leg in six adults during passive simulated jogging for five minutes in which temperatures fell and continued to fall during recovery for an additional ten minutes. The skin temperature was stable (Control) in a subject resting the feet on non-moving pedals. Panel B. . Skin temperatures of the shoulder in six adults during passive simulated jogging for five minutes in which temperatures fell and continued to fall during recovery for an additional ten minutes. This is consistent with a systemic effect.

\section{Interventions in the workplace and home to reduce physical inactivity}

There have been many devices and counselling techniques advocated for achieving the replacement of two to four hours of sitting a day with a physical activity but none have been highly successive for population groups in the short term (up to three months) or long term. The passive simulated jogging device described above is too new to be tested but preliminary acceptance has been high. The following lists examples of interventions along with selected commercial examples:

1. Sit-stand desk (VARIDESK, CoppellTX) alone or with counselling

2. Treadmill desk (NextDesk ${ }^{\mathrm{TM}}$, Georgetown, TX), alone or with counselling

3. Pedaling workstations (WeWatt, Leuven, Belgium)

4. Walking during breaks

5. Computer prompts to stand (Stir, Los Angeles, CA). These mechanical technologies often require multitasking whereas the passive simulated jogging device does not.

Clearly, there is urgent need to thwart this epidemic of obesity and Type 2 diabetes with awareness, education as well as non-invasive, wellness interventions that have a physiological basis. The passive simulated jogging device described in the paper is potentially one such solution but needs long-term evaluation of compliance.

\section{Acknowledgements}

None.

\section{Conflict of interest}

Dr. Sackner and Dr. Adams are co-founders of Sackner Wellness Products LLC and the co-inventors of the Passive Simulated Jogging Device.

\section{References}

1. Chu AH, Ng SH, Tan CS, et al. A systematic review and meta-analysis of workplace intervention strategies to reduce sedentary time in whitecollar workers. Obes Rev. 2016;17(5):467-481.

2. Duvivier BM, Schaper NC, Bremers MA, et al. Minimal intensity physical activity (standing and walking) of longer duration improves insulin action and plasma lipids more than shorter periods of moderate to vigorous exercise (cycling) in sedentary subjects when energy expenditure is comparable. Plos One. 2013;8(2):e55542.

3. Ekelund U, Steene Johannessen J, Brown WJ, et al. Does physical activity attenuate, or even eliminate, the detrimental association of sitting time with mortality? A harmonised meta-analysis of data from more than 1million men and women. Lancet. 2016;388(10051):1302-1310.

4. Hadgraft NT, Lynch BM, Clark BK, et al. Excessive sitting at work and at home: Correlates of occupational sitting and TV viewing time in working adults. BMC Public Health. 2015;15:899.

5. Holtermann A, Mork PJ, Nilsen TI. Hours lying down per day and mortality from all-causes and cardiovascular disease: the HUNT Study, Norway. Eur J Epidemiol. 2014;29(8):559-565.

6. Merla A, Mattei PA, Di DL, et al. Thermal imaging of cutaneous temperature modifications in runners during graded exercise. Ann Biomed Eng. 2010;38(1):158-163.

7. Morishima T, Restaino RM, Walsh LK, et al. Prolonged sitting-induced leg endothelial dysfunction is prevented by fidgeting. Am J Physiol Heart Circ Physiol. 2016;311(1):H177-H182. 
8. Ornstein KA, Leff B, Covinsky KE, et al. Epidemiology of the homebound population in the United States. JAMA Intern Med. 2015;175(7):1180-1186.

9. Padilla J, Olver TD, Thyfault JP, et al. Role of habitual physical activity in modulating vascular actions of insulin. Exp Physiol. 2015;100(7):759771 .

10. Peterman JE, Wright KP, Melanson EL, et al. Motor-Driven (Passive) Cycling: A Potential Physical Inactivity Countermeasure? Med Sci Sports Exerc. 2016;48(9):1821-1828.

11. Reynolds LJ, Credeur DP, Manrique C, et al. Obesity, type 2 diabetes, and impaired insulin-stimulated blood flow: role of skeletal muscle NO synthase and endothelin-1. J Appl Physiol (1985). 2017;122(1):38-47.
12. Sackner MA, Gummels E, Adams JA. Nitric oxide is released into circulation with whole-body, periodic acceleration. Chest. 2005;127(1):30-39.

13. Sackner MA, Gummels E, Adams JA. Effect of moderate-intensity exercise, whole-body periodic acceleration, and passive cycling on nitric oxide release into circulation. Chest. 2005;128(4):2794-2803.

14. Sakaguchi M, Fukuda S, Shimada K, et al. Preliminary observations of passive exercise using whole body periodic acceleration on coronary microcirculation and glucose tolerance in patients with type 2 diabetes. $J$ Cardiol. 2012;60(4):283-287. 